\title{
UNIFORM DISTRIBUTION OF SEQUENCES IN RINGS OF INTEGRAL QUATERNIONS
}

\author{
by L. KUIPERS and JAU-SHYONG SHIUE
}

(Received 15 May, 1980; revised 30 October, 1980)

1. Introduction. Let $\mathbb{Z}$ and $\mathbb{Z}[i]$ have their usual meaning. Let $Y_{0}$ denote the noncommutative ring of integral quaternions, that is the set of all elements $a+b i+c j+d k$ with $a, b, c, d \in \mathbb{Z}$ and where $i, j$ and $k$ together with the number 1 are the four units of the system of quaternions.

Let $\mathcal{N}(\mu)=a^{2}+b^{2}+c^{2}+d^{2}$ be the norm of the element $\mu=a+b i+c j+d k \in Y_{0}$. The nontrivial ideals in $Y_{0}$ are exactly the principal ideals $(\mu)$ generated by elements $\mu \in Y_{0}$ with $\mathcal{N}(\mu) \geqslant 2$.

Analogous to the definition of uniformly distributed sequences in $\mathbb{Z}$ due to Niven [4] (see also Kuipers and Niederreiter [1, Chapter 5]) and that in $\mathbb{Z}[i]$ due to Kuipers, Niederreiter and Shiue [2] we consider sequences of integral quaternions and ask how they are distributed modulo an arbitrary nontrivial left ideal in $Y_{0}$. We introduce the following definition.

DEFINITION 1. Let $\left(\mu_{n}\right)(n=1,2, \ldots)$ be a sequence of integral quaternions. Let $\beta, \mu \in Y_{0}$ with $\mathcal{N}(\mu) \geqslant 2$. For a positive integer $N$, let $A(\beta, \mu, N)$ denote the number of $n$ $(1 \leqslant n \leqslant N)$ such that $\mu_{n} \equiv \beta(\bmod \mu)$. The sequence $\left(\mu_{n}\right)$ is said to be uniformly distributed modulo $\mu$ (abbreviated u.d. $\bmod \mu$ ) in $Y_{0}$ if

$$
\lim _{N \rightarrow \infty} A(\beta, \mu, N) / N=(\mathcal{N}(\mu))^{-2}, \ldots
$$

for all $\beta \in Y_{0}$. (The choice of $\beta$ may be restricted to all elements of a complete residue system modulo $\mu$ (abbreviated c.r.s. $\bmod \mu$ )). The sequence $\left(\mu_{n}\right)$ is said to be u.d. in $Y_{0}$ if it is u.d. $\bmod \mu$ for all $\mu \in Y_{0}$ with $\mathcal{N}(\mu) \geqslant 2$.

When investigating specific sequences it is useful to have available an explicit description of a c.r.s. $\bmod \mu$.

Let $H(\gamma)$ denote a c.r.s. $\bmod \gamma(\gamma \in \mathbb{Z}[i])$ in $\mathbb{Z}[i]$. If $\mu=a+b i+c j+d k \in Y_{0}$, then we denote the g.c.d. (or one of its associates) of the elements $a+b i$ and $c-d i \in \mathbb{Z}[i]$ by $\delta=(a+b i, c-d i)$. The g.c.d. of the integers $a, b, c, d \in \mathbb{Z}$ is denoted by $g=(a, b, c, d)$. The conjugate of $\mu=a+b i+c j+d k \in Y_{0}$ is denoted by $\mu^{*}=a-b i-c j-d k$. We have

$$
\mu \mu^{*}=\mu^{*} \mu=a^{2}+b^{2}+c^{2}+d^{2}=\mathcal{N}(\mu) .
$$

The following description of a c.r.s. modulo $q$ was given by Shiue and Hwang [5].

THEOREM 1. A c.r.s. $\bmod q$, where $q=a+b i+c j+d k \in Y_{0}(q \neq 0)$ is given by the set

$$
G(q)=\left\{r_{0}+r_{1} i+r_{2} j+r_{3} k \mid r_{0}+r_{1} i \in H(\delta), r_{2}+r_{3} i \in H\left(\mathcal{N}(q) \delta^{-1}\right)\right\} .
$$

Here $\delta=(a+b i, c-d i)$. 
Corollary. A c.r.s. $\bmod q$, where $q=a+b i+c j+d k(q \neq 0)$, is given by the set

$$
\begin{aligned}
& G(q)=\left\{r_{0}+r_{1} i+r_{2} j+r_{3} k \mid 0 \leqslant r_{0}<\mathcal{N}(\delta) g^{-1}, 0 \leqslant r_{1}<g,\right. \\
& \left.0 \leqslant r_{2}<\mathcal{N}(q) \mathcal{N}(\delta)^{-1} g, 0 \leqslant r_{3}<\mathcal{N}(q) g^{-1}\right\} \text {. }
\end{aligned}
$$

The cardinality of $G(q)$ is $\mathcal{N}(q)^{2}$.

2. Uniform distribution modulo an ideal. The elements of a c.r.s. modulo a nontrivial (left) ideal form an additive group. With regard to this group we have the following result.

Theorem 2. For $\mu=a+b i+c j+d k \in Y_{0}$ with $\mu \neq 0, g=(a, b, c, d), \delta=(a+b i, c-d i)$, the characters of the group $Y_{0} /(\mu)$ are given by

$$
\begin{aligned}
\chi(x+y i+z j+u k)= & \exp \left\{\left(r_{0}(a x+b y+c z+d u)+r_{1}(-b x+a y+d z-c u)\right.\right. \\
& \left.\left.+r_{2}(-c x-d y+a z+b u)+r_{3}(-d x+c y-b z+a u)\right) / \mathcal{N}(\mu)\right\},
\end{aligned}
$$

where $0 \leqslant r_{0}<\mathcal{N}(\delta) g^{-1}, 0 \leqslant r_{1}<g, 0 \leqslant r_{2}<\mathcal{N}(\mu) \mathcal{N}(\delta)^{-1} g, 0 \leqslant r_{3}<\mathcal{N}(\mu) g^{-1}$, and where $\exp t$ stands for $e^{2 \pi i t}$ for real $t$.

Proof. First, these expressions are really characters of $Y_{0} /(\mu)$, for assume that $x+y i+z j+u k \equiv x_{0}+y_{0} i+z_{0} j+u_{0} k \quad(\bmod \mu)$. Then we have $\chi(x+y i+z j+u k)=$ $x\left(x_{0}+y_{0} i+z_{0} j+u_{0} k\right)$ if and only

$r_{0}(a x+b y+c z+d u)+\ldots+r_{3}(-d x+c y-b z+a u)$

$$
\equiv r_{0}\left(a x_{0}+b y_{0}+c z_{0}+d u_{0}\right)+\ldots+r_{3}\left(-d x_{0}+c y_{0}-b z_{0}+a u_{0}\right) \quad(\bmod \mathcal{N}(\mu)),
$$

a congruence which can be written in the form:

$$
\begin{aligned}
& r_{0}\left\{a\left(x-x_{0}\right)+b\left(y-y_{0}\right)+c\left(z-z_{0}\right)+d\left(u-u_{0}\right)\right\}+\ldots \\
& +r_{3}\left\{-d\left(x-x_{0}\right)+c\left(y-y_{0}\right)-b\left(z-z_{0}\right)+a\left(u-u_{0}\right)\right\} \equiv 0 \quad(\bmod \mathcal{N}(\mu)) .
\end{aligned}
$$

Now we have for some $A, B, C, D \in \mathbb{Z}$ the relation

$$
\left(x-x_{0}\right)+\left(y-y_{0}\right) i+\left(z-z_{0}\right) j+\left(u-u_{0}\right) k=(a+b i+c j+d k)(A+B i+C j+D k),
$$

which implies the system

$$
\begin{array}{ll}
x-x_{0}=a A-b B-c C-d D, & y-y_{0}=a B+b A+c D-d C, \\
z-z_{0}=a C-b D+c A+d B, & u-u_{0}=a D+b C-c B+d A,
\end{array}
$$

and therefore we have

$$
\begin{aligned}
a\left(x-x_{0}\right)+b\left(y-y_{0}\right)+c\left(z-z_{0}\right)+d\left(u-u_{0}\right) & =A \mathcal{N}(\mu), \\
-b\left(x-x_{0}\right)+a\left(y-y_{0}\right)+d\left(z-z_{0}\right)-c\left(u-u_{0}\right) & =B \mathcal{N}(\mu) \\
-c\left(x-x_{0}\right)-d\left(y-y_{0}\right)+a\left(z-z_{0}\right)+b\left(u-u_{0}\right) & =C \mathcal{N}(\mu), \\
-d\left(x-x_{0}\right)+c\left(y-y_{0}\right)-b\left(z-z_{0}\right)+a\left(u-u_{0}\right) & =D \mathcal{N}(\mu),
\end{aligned}
$$

and thus the above congruence is satisfied.

Second, for $\xi, \eta \in Y_{0}$, one has $\chi(\xi+\eta)=\chi(\xi) \chi(\eta)$. 
Third, the abelian group $Y_{0} /(\mu)$ has $\mathcal{N}(\mu)^{2}$ characters, so that it suffices to show that the $\mathcal{N}(\mu)^{2}$ characters $\chi$ given above are distinct.

Suppose that

$$
\begin{aligned}
r_{0}(a x+b y+c z+d u) & +\ldots+r_{3}(-d x+c y-b z+a u) \\
& \equiv s_{0}(a x+b y+c z+d u)+\ldots+s_{3}(-d x+c y-b z+a u) \quad(\bmod \mathcal{N}(\mu))
\end{aligned}
$$

for all $x+y i+z j+u k \in Y_{0}$, and where $r_{0}, r_{1}, r_{2}, r_{3}, s_{0}, s_{1}, s_{2}, s_{3}$ satisfy the abovementioned inequalities.

Now we substitute $x=-b / g, y=a / g, z=d / g, u=-c / g$. Then we have

$$
a x+b y+c z+d u=0,-c x-d y+a z+b u=0,-d x+c y-b z+a u=0
$$

and the last mentioned congruence reduces to $\left(r_{1}-s_{1}\right) \mathcal{N}(\mu) / g \equiv 0(\bmod \mathcal{N}(\mu))$, and since $\left|r_{1}-s_{1}\right|<g$ we must have $r_{1}=s_{1}$. Hence according to our assumption we have for all $x+y i+z j+u k \in Y_{0}$ the congruence

$$
\begin{aligned}
& r_{0}(a x+b y+c z+d u)+r_{2}(-c x-d y+a z+b u)+r_{3}(-d x+c y-b z+a u) \\
& \equiv s_{0}(a x+b y+c z+d u)+s_{2}(-c x-d y+a z+b u)+s_{3}(-d x+c y-b z+a u) \quad(\bmod \mathcal{N}(\mu)) .
\end{aligned}
$$

Now there are infinitely many numbers $x, y, z, u \in \mathbb{Z}$ such that

$$
a x+b y+c z+d u=g \mathcal{N}(\mu) / \mathcal{N}(\delta), \quad-c x-d y+a z+b u=0, \quad-d x+c y-b z+a u=0 .
$$

Upon substitution we obtain

$$
\left(r_{0}-s_{0}\right) \mathcal{N}(\mu) g / \mathcal{N}(\delta) \equiv 0 \quad(\bmod \mathcal{N}(\mu)),
$$

which is possible only if $r_{0}=s_{0}$ since $\left|r_{0}-s_{0}\right|<\mathcal{N}(\delta) / g$. Hence the congruence we are investigating reduces to

$$
\left(r_{2}-s_{2}\right)(-c x-d y+a z+b u)+\left(r_{3}-s_{3}\right)(-d x+c y-b z+a u) \equiv 0 \quad(\bmod \mathcal{N}(\mu)),
$$

valid for all $x+y i+z j+u k \in Y_{0}$. Now there are infinitely many integers $x, y, z, u \in \mathbb{Z}$ such that

$$
-c x-d y+a z+b u=\mathcal{N}(\delta) / g, \quad-d x+c y-b z+a u=0 .
$$

Then upon substitution one obtains the congruence

$$
\left(r_{2}-s_{2}\right) \mathcal{N}(\delta) / g \equiv 0 \quad(\bmod \mathcal{N}(\mu)) .
$$

Since $\left|r_{2}-s_{2}\right|<\mathcal{N}(\mu) g / \mathcal{N}(\delta)$, we must have $r_{2}=s_{2}$.

Finally assume that

$$
r_{3}(-d x+c y-b z+a u) \equiv s_{3}(-d x+c y-b z+a u) \quad(\bmod \mathcal{N}(\mu))
$$

for all $x+y i+z j+u k \in Y_{0}$. Now choose $\xi, \eta, \phi, \psi \in \mathbb{Z}$ such that $g=-d \xi+c \eta-b \phi+a \psi$. Then we obtain

or

$$
\left(r_{3}-s_{3}\right)(-d \xi+c \eta-b \phi+a \psi) \equiv 0 \quad(\bmod \mathcal{N}(\mu)),
$$

$$
\left(r_{3}-s_{3}\right) g \equiv 0 \quad(\bmod \mathcal{N}(\mu)) .
$$

But since $\left|r_{3}-s_{3}\right|<\mathcal{N}(\mu) / g$ we have $r_{3}=s_{3}$. 
Corollary. The characters of $Y_{0} /(\mu)$ are given by $\chi_{\theta}(\xi)=\exp \operatorname{Re}\left(\xi \theta^{*} / \mu\right)$, where

$$
\xi=x+y i+z j+u k, \quad \theta=r_{0}+r_{1} i+r_{2} j+r_{3} k \quad(\theta \in G(\mu)) .
$$

TheOREm 3. (Weyl criterion). The sequence $\left(x_{n}\right)\left(n=1,2, \ldots, x_{n} \in Y_{0}\right)$ is u.d. $\bmod \mu$ if and only if

$$
\lim _{N \rightarrow \infty} \frac{1}{N} \sum_{n=1}^{N} \exp \operatorname{Re}\left(x_{n} \theta^{*} / \mu\right)=0
$$

for all $\theta \in G(\mu)$.

Proof. By viewing $Y_{0} /(\mu)$ as a compact abelian group with the discrete topology the assertion follows immediately from the Weyl criterion on such groups (see [1, Chapter 4]) and from the above corollary.

THEOREM 4. Let $\mu \in Y_{0}$ with $\mathcal{N}(\mu) \geqslant 2$. Assume $\lambda \mid \mu$ where $\lambda \in Y_{0}$ with $\mathcal{N}(\lambda) \geqslant 2$. Then a sequence in $Y_{0}$ is u.d. $\bmod \lambda$ whenever it is u.d. $\bmod \mu$.

Proof. For any $\nu \in Y_{0}$ we have $A(\nu, \lambda, N)=\sum_{\omega+(\mu) \in S} A(\omega, \mu, N)$ where $S$ is the set of all distinct $\omega+(\mu)$ such that $\omega \equiv \nu(\bmod \lambda)$. The cardinality of $S$ is equal to the cardinality of $(\lambda) /(\mu)$. From the group isomorphism theorem we know that

$$
Y_{0} /(\lambda) \cong\left(Y_{0} /(\mu)\right) /((\lambda) /(\mu))
$$

and hence $\mathcal{N}(\lambda)^{2}=\mathcal{N}(\mu)^{2} / \operatorname{card}(\lambda) /(\mu)$, or

Hence

$$
\operatorname{card} S=\operatorname{card}(\lambda) /(\mu)=\mathcal{N}(\mu)^{2} / \mathcal{N}(\lambda)^{2} .
$$

$$
\begin{aligned}
\lim _{N \rightarrow \infty} A(\nu, \lambda, N) / N & =\lim _{N \rightarrow \infty} \sum_{\omega+(\mu) \in S}(A(\omega, \mu, N) / N) \\
& =\sum_{\omega+(\mu) \in S} \lim _{N \rightarrow \infty}(A(\omega, \mu, N) / N) \\
& =\operatorname{card} S . \mathcal{N}(\mu)^{-2} \\
& =\mathcal{N}(\lambda)^{-2} .
\end{aligned}
$$

THEOREM 5. Let $\mu_{1}, \mu_{2}, \ldots$ be a finite or denumerable collection of integral quaternions with $\mathcal{N}\left(\mu_{n}\right) \geqslant 2(n=1,2, \ldots)$. Then there exists a sequence in $Y_{0}$ which is $u . d$. $\bmod \mu_{n}$ for all $n$, but which is not u.d. $\bmod \lambda$ for $\lambda \in Y_{0}, \mathcal{N}(\lambda) \geqslant 2$ with $\mu_{n} \notin(\lambda)$ for all $n$.

Proof. We apply the following theorem of Zame [6]: Let $G$ be a locally compact abelian group with countable base, and let $\varphi \neq \varnothing$ and $\mathscr{T}$ be countable collections of closed subgroups of $G$ such that (i) finite intersections of members of $\varphi \cup \mathcal{T}$ are of compact index, (ii) for each $S \in \varphi$ and $T \in \mathscr{T}$ we have $S \nsubseteq T$, (iii) for each $T \in \mathscr{T}$ there exists a character $\chi_{T}$ of $G$ such that $\chi_{\mathrm{T}}$ is trivial on $T$ but is nontrivial on each $S \in \varphi$. Then there is a sequence $\left(g_{n}\right)$ $(n=1,2, \ldots)$ in $G$ such that $\left(g_{n}\right)$ is u.d. $\bmod S$ for all $S \in \varphi$, but not u.d. $\bmod T$ for $T \in \mathscr{T}$.

Now let $G=Y_{0}$ with the discrete topology, and let $\varphi$ consist of the left ideals $\left(\mu_{1}\right)$, 
$\left(\mu_{2}\right), \ldots$ whereas $\mathscr{T}$ consists of all nonzero left ideals $(\lambda), \mathcal{N}(\lambda) \geqslant 2$ with $\mu_{n} \notin(\lambda)$ for all $n$. Then conditions (i) and (ii) are easily checked. (Note that every nonzero left ideal in $Y_{0}$ has finite, thus compact index.) As to condition (iii), let $(\lambda) \in \mathscr{T}$ be given, and choose $\theta=1$ in the character formula for $Y_{0} /(\lambda)$. Then we get a character $\chi$ with $\chi(\xi)=\exp \operatorname{Re}(\xi / \lambda)$ for $\xi \in Y_{0}$. This character is trivial on $(\lambda)$. For any $n$ we have

$$
\mu_{n} / \lambda=a_{n}+b_{n} i+c_{n} j+d_{n} k \notin Y_{0},
$$

and the following statements hold:

$$
\begin{array}{llll}
\text { if } & a_{n} \notin \mathbb{Z} \text { then } \quad \chi\left(\mu_{n}\right)=\exp \operatorname{Re}\left(\mu_{n} \lambda^{-1}\right)=\exp a_{n} \neq 1 ; \\
\text { if } & b_{n} \notin \mathbb{Z} \text { then } \chi\left(i \mu_{n}\right)=\exp \operatorname{Re}\left(i \mu_{n} \lambda^{-1}\right)=\exp \left(-b_{n}\right) \neq 1 ; \\
\text { if } \quad c_{n} \notin \mathbb{Z} \text { then } \chi\left(j \mu_{n}\right)=\exp \operatorname{Re}\left(j \mu_{n} \lambda^{-1}\right)=\exp \left(-c_{n}\right) \neq 1 ; \\
\text { if } \quad d_{n} \notin \mathbb{Z} \text { then } \chi\left(k \mu_{n}\right)=\exp \operatorname{Re}\left(k \mu_{n} \lambda^{-1}\right)=\exp \left(-d_{n}\right) \neq 1 .
\end{array}
$$

In all cases $\chi$ is nontrivial on $\left(\mu_{n}\right)$. Thus $\chi$ satisfies the conditions in (iii) and the theorem follows immediately.

LemMA 1. Let $\mu=a+b i+c j+d k \in Y_{0}, \mu \neq 0, g=(a, b, c, d), h \in \mathbb{Z}$. Then $\mu \mid h$ if and only if $(\mathcal{N}(\mu) / g) \mid h$.

Proof. Write $a=g a_{1}, \ldots, d=g d_{1}$. Assume $(\mathcal{N}(\mu) / g) \mid h$, or $(a+b i+c j+d k)$. $\left(a_{1}-b_{1} i-c_{1} j-d_{1} k\right) \mid h$. Then $\mu \mid h$. Conversely let $\mu \mid h$. Then for some $C, D, E, F \in \mathbb{Z}$ we have

$$
\begin{aligned}
h= & (a+b i+c j+d k) .(C+D i+E j+F k)=a C-b D-c E-d F \\
& +(a D+b C+c F-d E) i+(a E-b F+c C+d D) j+(a F+b E-c D+d C) k .
\end{aligned}
$$

Hence

We obtain

$$
\left\{\begin{array}{l}
b C+a D-d E=-c F, \\
c C+d D+a E=b F, \\
d C-c D+b E=-a F,
\end{array} \text { and } \quad \operatorname{det}\left[\begin{array}{rrr}
b & a & -d \\
c & d & a \\
d & -c & b
\end{array}\right]=d \mathcal{N}(\mu)\right.
$$

$$
d C=-F a, \quad d D=F b, \quad d E=F c .
$$

Since $h=a C-b D-c E-d F$, we have $d h=-\mathcal{N}(\mu) F$. Now there are three more similar relations. Since $\mathrm{g}=(a, b, c, d)$ there are integers $\xi, \eta, \zeta, \varepsilon \in \mathbb{Z}$ such that $\mathrm{g}=$ $\xi a+\eta b+\zeta c+\varepsilon d$. By addition we find that $(\mathcal{N}(\mu) / g) \mid h$.

THEOREM 6. Let $\mu=a+b i+c j+d k, \mathcal{N}(\mu) \geqslant 2$. Let $\left(h_{n}\right)(n=1,2, \ldots)$ be a sequence in $\mathbb{Z}$, and let $\alpha \in Y_{0}$. Then the sequence $\left(h_{n} \alpha\right)(n=1,2, \ldots)$ is not u.d. $\bmod \mu$.

Proof. Let $\left(h_{n} \alpha\right)$ by u.d. $\bmod \mu$. Then the residue classes of $\left(h_{n} \alpha\right) \bmod \mu$ must run through all elements of $Y_{0} /(\mu)$. In particular, the residue class of $\alpha \bmod \mu$ generates $Y_{0} /(\mu)$, so that $Y_{0} /(\mu)$ is cyclic. By Lemma 1 , we have $\beta \mathcal{N}(\mu) / g \equiv 0(\bmod \mu)$ for all $\beta \in Y_{0}$, and so the order of any residue class $\bmod \mu$ in $Y_{0} /(\mu)$ is at most $\mathcal{N}(\mu) / g$. Since $Y_{0} /(\mu)$ is cyclic of order $\mathcal{N}(\mu)^{2}$ we obtain a contradiction. 
Lemma 2. Let $\mu=a+b i+c j+d k \in Y_{0}, g=(a, b, c, d)$. Let $C+D i \in \mathbb{Z}[i]$. Then $(\mathcal{N}(\mu) / g) \mid C+$ Di implies $\mu \mid C+$ Di. If moreover $\left(a_{1}-b_{1} i, c_{1}-d_{1} i\right)=1$, where $a=g a_{1}, \ldots$, $d=g d_{1}$, then $\mu \mid C+D i$ implies $(\mathcal{N}(\mu) / g) \mid C+D i$.

Proof. The first implication to be shown is evident since

$$
\mathcal{N}(\mu) / g=\mu\left(a_{1}-b_{1} i-c_{1} j-d_{1} k\right) .
$$

Now assume $\mu \mid C+D i$. Hence for some $X, Y, Z, U \in \mathbb{Z}$ we have

$$
\begin{aligned}
C+D i & =(a+b i+c j+d k)(X+Y i+Z j+U k) \\
& =\{a+b i+j(c-d i)\} \cdot\{X+Y i+j(Z-U i)\} \\
& =(a+b i)(X+Y i)-(c+d i)(Z-U i)+j\{(c-d i)(X+Y i)+(a-b i)(Z-U i)\} .
\end{aligned}
$$

Hence

$$
\left(c_{1}-d_{1} i\right)(X+Y i)+\left(a_{1}-b_{1} i\right)(Z-U i)=0 .
$$

Let $a_{1}-b_{1} i$ and $c_{1}-d_{1} i$ be relatively prime. Then $a_{1}-b_{1} i \mid X+Y i$. Hence for some $s, t \in \mathbb{Z}$ we have $X+Y i=\left(a_{1}-b_{1} i\right)(s+t i)$. This implies that $Z-U i=-\left(c_{1}-d_{1} i\right)(s+t i)$. Substitution yields now that

$$
\begin{aligned}
C+D i & =(a+b i)\left(a_{1}-b_{1} i\right)(s+t i)+(c+d i)\left(c_{1}-d_{1} i\right)(s+t i) \\
& =\mathcal{N}(\mu)(s+t i) / g
\end{aligned}
$$

or $(\mathcal{N}(\mu) / g) \mid C+D i$.

THeOREM 7. Let $\mu=a+b i+c j+d k \in Y_{0}$ with $\mathcal{N}(\mu) \geqslant 2$, and let $(c-d i, \mathcal{N}(\mu))=1$ in $\mathbb{Z}[i]$. Let $p, q \in \mathbb{Z}$ be chosen such that in $\mathbb{Z}[i](c-d i)(p+q i) \equiv 1(\bmod \mathcal{N}(\mu))$. Then the sequence $\left(x_{n}+y_{n} i+z_{n} j+u_{n} k\right)(n=1,2, \ldots)$ is $u . d . \bmod \mu$ in $Y_{0}$ if and only if the sequence $\left(\chi_{n}\right)$, with

$$
\chi_{n}=x_{n}+y_{n} i-(p+q i)(a+b i)\left(z_{n}-u_{n} i\right)
$$

is u.d. $\bmod \mathcal{N}(\mu)$ in $\mathbb{Z}[i]$.

Proof. Since $(c-d i, \mathcal{N}(\mu))=1$, we have $g=(a, b, c, d)=1$, and also $(c-d i, a-b i)=1$. Hence we may apply Lemma 2 . Assume that the sequence $\left(\chi_{n}\right)$ is u.d. $\bmod \mathcal{N}(\mu)$ in $\mathbb{Z}[i]$; then $\left(\chi_{n}\right)$ is also u.d. $\bmod \mu$ in $Y_{0}$. For according to Lemma 2 we have the equivalence of the congruences $X \equiv Y(\bmod \mathcal{N}(\mu))$ and $X \equiv Y(\bmod \mu)$, where $X$ and $Y$ are in $\mathbb{Z}[i]$. Now we make use of the congruences

$$
\begin{aligned}
(c-d i)(p+q i) & \equiv 1(\bmod \mathcal{N}(\mu))(\text { our assumption) } \\
(c-d i)(p+q i) & \equiv 1(\bmod \mu)(\text { Lemma } 2) \\
-(a+b i) & \equiv j(c-d i)(\bmod \mu)
\end{aligned}
$$

and obtain

$$
-(a+b i)(p+q i) \equiv j(\bmod \mu)
$$


So the conclusion that

$$
\left(x_{n}+y_{n} i+j\left(z_{n}-u_{n} i\right)\right)=\left(x_{n}+y_{n} i+z_{n} j+u_{n} k\right)
$$

is u.d. $\bmod \mu$ is true. The converse can be shown similarly.

REMARK. The following statement is closely related to Theorem 7 and can be shown along the same lines. Let $\mu=a+b i+c j+d k \in Y_{0}, \mathcal{N}(\mu) \geqslant 2$. Assume $\delta=(a+b i, c-d i)=1$ in $\mathbb{Z}[i]$. Choose $\gamma \in \mathbb{Z}[i]$ such that

$$
\gamma(c-d i) \equiv 1(\bmod \mathcal{N}(\mu))
$$

in $\mathbb{Z}[i]$. Then for $\alpha_{n}, \beta_{n} \in \mathbb{Z}[i]$ the sequence $\left(\alpha_{n}+\beta_{n} j\right)(n=1,2, \ldots)$ is u.d. $\bmod \mu$ if and only if the sequence $\left(\alpha_{n}-\beta_{n} \gamma^{*}(a+b i)\right)$ is u.d. $\bmod \mathcal{N}(\mu)$ in $\mathbb{Z}[i]$.

3. Uniform distribution in $Y_{0}$. We recall the following definition: The sequence $\left(\left(x_{n}, y_{n}, z_{n}, u_{n}\right)\right)$ is u.d. in $\mathbb{Z}^{4}$ if it is u.d. modulo all subgroups of $\mathbb{Z}^{4}$ of finite index.

THEOREM 8. The sequence $\left(x_{n}+y_{n} i+z_{n} j+u_{n} k\right) \in Y_{0}(n=1,2, \ldots)$ is u.d. in $Y_{0}$ if and only if the sequence $\left(\left(x_{n}, y_{n}, z_{n}, u_{n}\right)\right)$ of lattice points is u.d. in $\mathbb{Z}^{4}$.

Proof. Let $\psi: Y_{0} \rightarrow \mathbb{Z}^{4}$ be the group isomorphism given by

$$
\psi(x+y i+z j+u k)=(x, y, z, u)
$$

for $x+y i+z j+u k \in Y_{0}$. The isomorphism $\psi$ maps the nontrivial ideals of $Y_{0}$ into subgroups of $\mathbb{Z}^{4}$ of finite index. Now let the sequence $\left(\left(x_{n}, y_{n}, z_{n}, u_{n}\right)\right)$ be u.d. in $\mathbb{Z}^{4}$. In particular, the sequence $\left(\left(x_{n}, y_{n}, z_{n}, u_{n}\right)\right)$ is then u.d. modulo all subgroups of $\mathbb{Z}^{4}$ corresponding to the nontrivial ideals of $Y_{0}$ under the isomorphism $\psi$, and therefore the sequence $\left(x_{n}+y_{n} i+z_{n} j+u_{n} k\right)$ is u.d. in $Y_{0}$.

To show the converse it suffices to prove that every subgroup of $\mathbb{Z}^{4}$ of finite index contains a subgroup corresponding to a nonzero ideal in $Y_{0}$ under the isomorphism $\psi$. So let $H$ be an arbitrary subgroup of $\mathbb{Z}^{4}$ of finite index. Let $h$ be the exponent of the factor group $\mathbb{Z}^{4} / H$, that is the smallest positive integer $h$ for which $h(a, b, c, d) \in H$ for all $(a, b, c, d) \in \mathbb{Z}^{4}$. Then we have

$$
h(a, b, c, d)=(h a, h b, h c, h d) \in H
$$

for all $(a, b, c, d) \in \mathbb{Z}^{4}$, so that

$$
H \supset h \mathbb{Z} \oplus h \mathbb{Z} \oplus h \mathbb{Z} \oplus h \mathbb{Z}
$$

Since the subgroup $h \mathbb{Z} \oplus h \mathbb{Z} \oplus h \mathbb{Z} \oplus h \mathbb{Z}$ corresponds to the principal ideal $(h)$ of $Y_{0}$ under the isomorphism $\psi$, the proof is complete.

THEOREM 9. Let $\left(x_{n}\right)$ be a sequence in $Y_{0}$ with

$$
x_{n}=a_{n}+b_{n} i+c_{n} j+d_{n} k \quad(n=1,2, \ldots) .
$$


Then $\left(x_{n}\right)$ is u.d. in $Y_{0}$ if and only if

$$
\lim _{N \rightarrow \infty} \frac{1}{N} \sum_{n=1}^{N} \exp \left(r_{1} a_{n}+r_{2} b_{n}+r_{3} c_{n}+r_{4} d_{n}\right)=0
$$

for all $r_{1}, r_{2}, r_{3}, r_{4} \in \mathbb{Q}$, not all four being integers.

Proof. This follows from Theorem 8 and the Weyl criterion for u.d. in $\mathbb{Z}^{4}$ given by Niederreiter [3].

THEOREM 10. The sequence $\left(\alpha_{n}\right)$ in $Y_{0}$ is u.d. in $Y_{0}$ if and only if for any $\xi=$ $a+b i+c j+d k \in Y_{0}$ with $(a, b, c, d)=1$ in $\mathbb{Z}$, the sequence $\left(\operatorname{Re}\left(\alpha_{n} \xi\right)\right)$ is u.d. in $\mathbb{Z}$.

Proof. Necessity. If $\alpha_{n}=x_{n}+y_{n} i+z_{n} j+u_{n} k$, we have

$$
\operatorname{Re}\left(\alpha_{n} \xi\right)=a x_{n}-b y_{n}-c z_{n}-d u_{n} .
$$

Assume that the sequence $\left(\alpha_{n}\right)$ is u.d. in $Y_{0}$. Then we have

$$
\lim _{N \rightarrow \infty} \frac{1}{N} \sum_{n=1}^{N} \exp \left(r_{1} x_{n}+r_{2} y_{n}+r_{3} z_{n}+r_{4} u_{n}\right)=0
$$

for all $r_{1}, r_{2}, r_{3}, r_{4} \in \mathbb{Q}$, not all four of them in $\mathbb{Z}$. Choose $r_{1}=a h / m, r_{2}=-b h / m, r_{3}=-c h / m$, $r_{4}=-d h / m(m \in \mathbb{Z}, m \geqslant 2, h=1,2, \ldots, m-1$.) So

$$
\lim _{N \rightarrow \infty} \frac{1}{N} \sum_{n=1}^{N} \exp \left(\left(a x_{n}-b y_{n}-c z_{n}-d u_{n}\right) h / m\right)=0 .
$$

Hence the sequence $\left(a x_{n}-b y_{n}-c z_{n}-d u_{n}\right)$ is u.d. in $\mathbb{Z}$.

Sufficiency. Let $r_{1}, r_{2}, r_{3}, r_{4}$ be four rational numbers, not all in $\mathbb{Z}$. We write $r_{1}=$ $R_{1} / S_{1}, r_{2}=R_{2} / S_{2}, r_{3}=R_{3} / S_{3}, R_{4}=R_{4} / S_{4}$, where $R_{1}, \ldots, R_{4}, S_{1}, \ldots, S_{4} \in \mathbb{Z}, S_{1}, \ldots, S_{4} \geqslant$ $1,\left(R_{1}, S_{1}\right)=1, \ldots,\left(R_{4}, S_{4}\right)=1$. Let $S=\left[S_{1}, S_{2}, S_{3}, S_{4}\right]$. Then $r_{1}=T_{1} / S, \ldots, r_{4}=T_{4} / S$. Hence

$$
r_{1} x_{n}+r_{2} y_{n}+r_{3} z_{n}+r_{4} u_{n}=\left(T_{1} x_{n}+T_{2} y_{n}+T_{3} z_{n}+T_{4} u_{n}\right) / S .
$$

Let $T_{1} \equiv V_{1}(\bmod S), \ldots, T_{4} \equiv V_{4}(\bmod S)\left(0 \leqslant V_{1}, \ldots, V_{4} \leqslant S-1\right)$. Let $h=\left(V_{1}, V_{2}, V_{3}, V_{4}\right)$ and write $V_{i}=h U_{i}$. Then $1 \leqslant h \leqslant S-1$ and

$$
\exp \left(r_{1} x_{n}+\ldots+r_{4} u_{n}\right)=\exp \left(\left(V_{1} x_{n}+\ldots+V_{4} u_{n}\right) / S\right)
$$

and moreover

$$
\begin{aligned}
V_{1} x_{n}+\ldots+V_{4} u_{n} & =\operatorname{Re}\left(x_{n}+y_{n} i+z_{n} j+u_{n} k\right)\left(U_{1}-U_{2} i-U_{3} j-U_{4} k\right) \\
& =\operatorname{Re}\left(\alpha_{n} \xi \mid \mu\right)
\end{aligned}
$$

with $\mu=1, \xi=U_{1}-U_{2} i-U_{3} j-U_{4} k$. According to our assumption we have

$$
\lim _{N \rightarrow \infty} \frac{1}{N} \sum_{n=1}^{N} \exp \left(\operatorname{Re}\left(\alpha_{n} \xi\right) / S\right)=0
$$


and so

$$
\lim _{N \rightarrow \infty} \frac{1}{N} \sum_{n=1}^{N} \exp \left(r_{1} x_{n}+r_{2} y_{n}+r_{3} z_{n}+r_{4} u_{n}\right)=0 .
$$

ExAmple. Let $\theta_{1}, \theta_{2}, \theta_{3}, \theta_{4}$ be real numbers such that $1, \theta_{1}, \theta_{2}, \theta_{3}, \theta_{4}$ are linearly independent over the rationals. Set $\alpha_{n}=\left[n \theta_{1}\right], \beta_{n}=\left[n \theta_{2}\right], \gamma_{n}=\left[n \theta_{3}\right], \delta_{n}=\left[n \theta_{4}\right]$. According to Niederreiter [3] the sequence $\left(\left(\alpha_{n}, \beta_{n}, \gamma_{n}, \delta_{n}\right)\right)$ is u.d. in $\mathbb{Z}^{4}$, and hence the sequence $\left(\alpha_{n}+\beta_{n} i+\gamma_{n} j+\delta_{n} k\right)$ is u.d. in $Y_{0}$.

We wish to thank the referee for suggested improvements.

\section{REFERENCES}

1. L. Kuipers and H. Niederreiter, Uniform distribution of sequences (Wiley-Interscience, New York, 1974).

2. L. Kuipers, H. Niederreiter, and J.-S. Shiue, Uniform distribution of sequences in the ring of Gaussian integers, Bull. Inst. Math. Acad. Sinica 3 (1975), 311-325.

3. H. Niederreiter, On a class of sequences of lattice points, J. Number Theory 4 (1972), 477-502.

4. I. Niven, Uniform distribution of sequences of integers, Trans. Amer. Math. Soc. 98 (1961), $52-61$

5. J.-S. Shiue and C.-P. Hwang, A note on a complete residue system in the ring of integral quaternions, Soochow J. Math. Natur. Sci. 5 (1979), 193-196.

6. A. Zame, On a problem of Narkiewicz concerning uniform distributions of sequences of integers, Colloq. Math. 24 (1972), 271-273.

DEPARTMENT OF MATHEMATICS

SOUTHERN ILLINOIS UNIVERSITY

Carbondale, Illinois 62901

U.S.A.
Department of Mathematical Sciences National ChengChi University TAIPEI, TAIWAN

R.O.C. 